\title{
EFEITOS DE SISTEMAS DE CULTIVO NA DENSIDADE E MACROPOROSIDADE DO SOLO E NO DESENVOLVIMENTO RADICULAR DO MILHO EM LATOSSOLO ROXO'
}

\author{
PAULO CÉSAR CORSINI² e ANTONIO SERGIO FERRAUDO ${ }^{3}$
}

\begin{abstract}
RESUMO - Neste trabalho foram estudados os efeitos imediato e residual de dois sistemas de preparo na densidade e macroporosidade do solo e no desenvolvimento radicular do milho (Zea mays L.), em camadas estruturalmente estabilizadas de um Latossolo Roxo, mantido por longo período sob plantio direto de milho. Os efeitos imediatos das operações envolvendo a subsolagem e a aração e gradagem aumentaram, em menos de um ano agrícola, a macroporosidade da camada superficial desse solo bem como o potencial de desenvolvimento radicular. Nesses tratamentos e nos três primeiros anos agrícolas, a adoção contínua do sistema de plantio direto diminuiu a porosidade de aeração do solo e o potencial de desenvolvimento radicular do milho. Os benefícios da manutenção desse sistema conservacionista nos valores de macroporosidade e densidade na camada superficial do solo iniciaram-se no quarto ano agrícola. A partir daí aumentaram, atingindo no oitavo ano agrícola consecutivo valores semelhantes aos imediatamente obtidos após as operações mecânicas realizadas na instalação do experimento. As relações entre desenvolvimento radicular, densidade e macroporosidade do solo foram estabelecidas por equações bem como por classes de desenvolvimento radicular.
\end{abstract}

Termos para indexação: Zea mays, porosidade, estrutura do solo, plantio direto.

\begin{abstract}
EFFECTS OF TILLAGE SYSTEMS ON BULK DENSITY, AERATION POROSITY AND ROOT DEVELOPMENT OF CORN IN A TYPIC HAPLORTHOX SOIL
\end{abstract}

\begin{abstract}
The objective of this study was to evaluate the immediate and the residual effects of soil preparation on bulk density, aeration porosity and root development relationships in stabilized structural layers of a typic Haplorthox soil due to long-term no-tillage system of corn (Zea mays L.).The immediate effects of soil preparation to planting involving subsoiling, plowing, and disking improved soil macroporosity and root development for a short period of time. In these treatments and on the first three consecutive years, the adoption of continuous no-tillage management decreased soil macroporosity and root development. The long-term benefits of continuous no-tillage on soil macroporosity initiated at the fourth agricultural year. From there on these values increased and had returned to corresponding levels of immediate effects of mechanical tillage performed in the experimental area at the eighth consecutive agricultural year. The relationships between root development, bulk density and macroporosity were established by equations and as a range of root development classes.
\end{abstract}

Index terms: Zea mays, porosity, soil structure, no-tillage.

\section{INTRODUÇÃO}

A presença de uma estrutura maciça adensada nas camadas superficial e subsuperficial é comum

\footnotetext{
${ }^{1}$ Aceito para publicação em 17 de abril de 1998.

${ }^{2}$ Eng. Agr., Prof. Titular, Dep. de Solos e Adubos, FCAVJUNESP, CEP 14870-000 Jaboticabal, SP. Bolsista do CNPq. E-mail: pcorsini@fcav.unesp.br

${ }^{3}$ Matemático, Dr., Prof. Assist., Dep. de Ciências Exatas, FCAVJ-UNESP.
}

no Latossolo Roxo cultivado intensivamente e a bibliografia pertinente tem demonstrado que nelas os valores de densidade do solo são mais elevados, a aeração é prejudicada bem como a penetração e a proliferação de raízes de milho. Para solucionar o problema de forma imediata, os agricultores têm utilizado como rotina de preparação do solo para o plantio a subsolagem precedendo a outras operações convencionalmente utilizadas (Castro Filho et al., 1993). Essa operação quando realizada com 
umidade acima do ponto de sazão resulta em fendas pouco maiores que os órgãos ativos do subsolador (Cooper, 1971). No solo seco resulta num conjunto de fendas e trincas de formas triangulares (Trouse Junior \& Humbert, 1959), com aumento da infiltração vertical da água no solo e lixiviação de sais solúveis em profundidade (Alzubaidi \& Webster, 1982). O sucesso dessa operação, entretanto, depende da permanência do seu efeito nas condições estruturais desse solo, relacionadas ao estado de dispersão dos colóides, composição, condições climáticas e sistemas de cultivo empregados. De maneira geral, os resultados não têm sido satisfatórios do ponto de vista da melhoria das condições físicas no Latossolo Roxo (Casagrande et al., 1975, 1981; Corsini, 1991) e poucas são as informações sobre tais efeitos no desenvolvimento radicular do milho. De acordo com Corsini (1993), quanto mais degradada a estrutura natural do solo maior a necessidade de mobilização, e quanto mais mobilizado pior a sua estrutura.

Se o cultivo intensivo é responsável pela degradação da estrutura do solo, facilitando a ação dos elementos do clima, homem e máquinas (Johnston et al., 1942; Marques, 1949; Corsini, 1974; Davidson et al., 1977; Vieira, 1981; Dalla Rosa, 1984; Chan \& Mead, 1988; Freebairn et al., 1993), sua redução com o acúmulo de resíduos orgânicos na superfície do solo provavelmente irá reverter tal situação (Shear \& Moscher, 1969; Triplett Junior \& Van Doren Junior, 1969; Blevins et al., 1977, 1978; Muzzili, 1983; Vieira \& Muzzili, 1984; Corsini, 1988; Chan et al., 1992). Segundo Dexter (1988), as práticas naturais podem ser mais satisfatórias que o preparo mecânico do solo para melhorar sua estrutura.

A técnica de plantio direto tem sido preconizada como uma alternativa para evitar os efeitos indesejáveis do preparo do solo, realizado de forma repetitiva e inadequada. Entretanto, não se pode esperar que o efeito do cultivo intensivo por diversos anos seja resolvido de imediato com a adoção de tal prática, uma vez que fatores como adequação à cultura, ao solo, às condições climáticas regionais e aos fatores naturais devem ser levados em conta.

Tratando-se de solos argilosos, um dos aspectos a ser estudado relaciona-se à estrutura, cuja importância, do ponto de vista do desenvolvimento radicular das plantas, associa-se à estabilidade, continuidade dos poros, porosidade total e distribuição dos poros pelo tamanho (Hamblin, 1987; Lal, 1993). A estabilidade estrutural envolve estudos de resistência à alteração natural ou induzida na arquitetura do solo e de continuidade dos poros. Esta, por sua vez, envolve estudos de dispersibilidade, migração e obstrução de poros por partículas finas e sua relação com a permeabilidade do solo à água e ao ar.

A porosidade total e a distribuição dos poros pelo tamanho, características físicas do solo indiretamente relacionadas à estrutura, podem ser avaliadas em termos de densidade e macroporosidade do solo. Segundo Douglas (1986) e Carter (1988), a macroporosidade revela-se como um índice bastante útil na avaliação das modificações estruturais do solo. Entretanto, reflete mais a condição do solo no momento e local da amostragem do que uma condição final ou permanente. Por essa razão, Pidgeon \& Soane (1977) e Carter \& Kunelius (1986) expressaram a macroporosidade e a densidade do solo como porcentagem de algum valor padrão, no estado de estabilidade estrutural (Kay et al., 1988), que corresponde ao terceiro estádio de degradação do solo (Corsini, 1991). Níveis ótimos e críticos de aeração, em relação ao desenvolvimento radicular, foram estabelecidos por alguns autores, como Greenland (1981), apesar da complexidade das relações existentes e de sua variação espacial e temporal no solo, na planta e no clima.

Objetivou-se neste trabalho avaliar os efeitos imediato e residual de dois sistemas de preparo do solo na densidade e macroporosidade do solo e no desenvolvimento radicular num Latossolo Roxo, ao longo de oito anos agrícolas consecutivos em que ficou sob plantio direto de milho.

\section{MATERIAL E MÉTODOS}

O experimento desenvolveu-se de 1987 a 1995 na Fazenda de Ensino e Pesquisa da Faculdade de Ciências Agrárias e Veterinárias, em Jaboticabal, São Paulo, em um Latossolo Roxo epieutrófico, A moderado, textura argilosa e intensivamente cultivado.

Os tratamentos consistiram em dois sistemas de manejo normalmente utilizados pelos agricultores: preparo convencional com aração e gradagem (C); e subsolagem, precedendo a aração e gradagem (SC). Incluiu-se um trata- 
mento referencial em volume de solo de $2 \times 2 \mathrm{~m}$ de superfície e $1 \mathrm{~m}$ de profundidade, denominado MAX-1, em que teoricamente as propriedades físicas, químicas e morfoestruturais do solo não limitam o potencial de produção máxima da cultura nas condições climáticas da região. A seguir, por oito anos agrícolas consecutivos, adotou-se o sistema de plantio direto de milho, em $\mathrm{C}$ e em SC.

As operações necessárias à instalação dos tratamentos iniciaram-se em 21 de setembro de 1987, com a realização, no solo seco, de uma subsolagem normal à curva de nível do terraço, numa profundidade de aproximadamente $40 \mathrm{~cm}$, empregando-se um trator Valmet 118-4, de $6950 \mathrm{~kg}$ e $122 \mathrm{CV}$ e um subsolador de três hastes fixas reguladas para espaçamento de plantio de milho, de $698 \mathrm{~kg}$. No dia 2 de outubro, realizou-se a aração normal às curvas de nível do terraço, com umidade do solo adequada (consistência friável) na área subsolada e na sem subsolagem. Nessa operação foram utilizados um trator Valmet 88 de $4052 \mathrm{~kg}$ e $81 \mathrm{CV}$ e um arado reversível de discos de 23" de diâmetro. No dia 15 de novembro de 1987, dois dias antes do plantio, procedeu-se a gradagem de nivelamento. Nos anos seguintes, as operações agrícolas resumiram-se à eliminação do mato com enxada rotativa, regulada para trabalho superficial $(88 / 89,89 / 90$ e 90/91), com herbicida (91/92, 92/93, 93/94 e 94/95) e plantio direto de milho (cultivar Braskalb 678 XL). Até o ano agrícola 91/92 o plantio foi realizado com "rotacaster", e após com plantadeiras nacionais, especialmente projetadas para efetuar o plantio de precisão no sistema de plantio direto. Essa operação foi realizada no início de cada ano agrícola, cujo primeiro ocorreu em 17/11/87.

A condução da cultura, envolvendo as operações de adubação de plantio e de cobertura, controle da lagartado-cartucho e outras, foi realizada de modo semelhante à da área cultivada convencionalmente pelo Setor de Produção da Faculdade, exceção feita, a partir do ano agrícola $88 / 89$, ao uso de máquinas agrícolas e à maneira de aplicação do corretivo, que seguiram as recomendações técnicas para o plantio direto.

Para a caracterização inicial do solo na área experimental, envolvendo $\mathrm{C}$ e $\mathrm{SC}$, antes da mobilização estabelecida experimentalmente (AM), foram coletados volumes não deformados de solo (54 mm de diâmetro, $60 \mathrm{~mm}$ de altura), utilizando-se um esquema sistemático (Petersen \& Calvin, 1986) e um amostrador tipo Uhland. Essa caracterização em termos de densidade e macroporosidade do solo ocorreu ao final do período de pousio do ano agrícola 86/87, com o solo apresentando, nas camadas superficial e subsuperficial, estrutura maciça, consistência dura e porosidade textural (Guerif, 1984), características do terceiro estádio do processo de degradação (Corsini, 1991). As amostras foram retiradas de $10 \mathrm{em}$ $10 \mathrm{~cm}$ até a profundidade de $80 \mathrm{~cm}$. Ao final do período de pousio relativo ao ano agrícola $94 / 95$, repetiu-se o procedimento para caracterização do solo. As amostras foram retiradas em $\mathrm{C}$ e SC e na linha (L) e entrelinha (EL) da cultura.

Os efeitos imediatos dos sistemas de preparo do solo na densidade e macroporosidade foram estabelecidos comparando-se os valores obtidos em AM com os avaliados em volumes não deformados de solo retirados logo após a mobilização (DM) em C e SC. Os efeitos residuais foram avaliados em amostragens realizadas durante o ciclo da cultura, três vezes em cada ano agrícola e mais uma vez cerca de um mês antes do plantio relativo ao ano seguinte. Os volumes de solo foram retirados em triplicatas nas camadas de 0-6 cm, 20-26 cm e 35-41 cm de profundidade, em L, EL, C e SC, exceção feita ao período 1987/91, cujas amostras foram retiradas somente na entrelinha da cultura.

Nos volumes de solo amostrados, os valores de densidade (Ds- $\mathrm{kg} \mathrm{dm}^{-3}$ ) foram avaliados pelo método do anel volumétrico (Blake, 1965) e os de macroporosidade $\left(\mathrm{S}-\mathrm{cm}^{3} \mathrm{~cm}^{-3}\right)$, da relação $\mathrm{S}=\alpha-\theta$, onde $\alpha\left(\mathrm{cm}^{3} \mathrm{~cm}^{-3}\right)$ é a porosidade total, calculada pela relação $\alpha=1$-(Ds/Dr), onde $\operatorname{Dr}\left(\mathrm{kg} \mathrm{dm}^{-3}\right)$ é a densidade real e $\theta\left(\mathrm{cm}^{3} \mathrm{~cm}^{-3}\right)$ é o teor de água retido no volume de solo quando submetido a um potencial matricial de $-60 \mathrm{~cm}$ de coluna d'água (Vomocil, 1965). Os valores de Dr foram avaliados pelo método do balão volumétrico (Embrapa, 1979).

Os efeitos na densidade do solo e na macroporosidade foram avaliados por meio da análise de variância e os fatores de variação consistiram de ano agrícola, tratamentos (C e SC), posição de amostragem (L e EL), camadas de solo (superficial, subsuperficial e de $35-41 \mathrm{~cm}$ ), condições de amostragem (AM e DM) e as interações duas a duas. O comportamento de Ds e S, ao longo do tempo, foi avaliado utilizando-se análises de regressão.

A variabilidade percentual da presença de raízes de milho visualizadas em perfis culturais (Tardieu \& Manichon, 1986), abertos em C e SC, na área sob plantio direto, na convencionalmente utilizada pelo Setor de Produção da Faculdade e no tratamento de máxima produção (MAX-1), ao final do ano agrícola $94 / 95$, orientou a retirada de volumes de solo não deformados, nas camadas superficial e subsuperficial. A partir deles, determinaramse os valores de Ds, $\mathrm{S}$ e a porcentagem relativa de comprimento radicular $(\mathrm{R} \%)$, onde $\mathrm{R} \%=$ (comprimento radicular do milho sob plantio direto e convencional/comprimento radicular do milho no tratamento MAX-1)x100. O comprimento radicular foi avaliado nas raízes de milho conti- 
das no volume de solo amostrado, seguindo método estabelecido por Tennant, (1975). Elas foram separadas do solo com peneiras de $1 \mathrm{~mm}$ de diâmetro médio de malha e jatos de água. Os valores encontrados foram utilizados para relacionar essas características, por regressões lineares e exponenciais, bem como definir classes de desenvolvimento radicular com o auxílio da técnica de análise de agrupamento (Sneath \& Sokal, 1973).

\section{RESULTADOS E DISCUSSÃO}

Nos volumes de solo amostrados nos perfis culturais, ao término do ano agrícola 94/95, os valores de densidade (Ds) correlacionaram-se aos de macroporosidade (S) de acordo com as equações: $\mathrm{Ds}=1,703-2,486 \mathrm{~S}\left(\mathrm{R}^{2}=0,97\right)$ na camada superficial e Ds $=1,515-1,472 \mathrm{~S},\left(\mathrm{R}^{2}=0,91\right)$ na camada subsuperficial. Pelas relações: $\mathrm{R} \%=4,850+$ $\exp (10,140-6,069 \mathrm{Ds})\left(\mathrm{R}^{2}=0,98\right)$ e $\mathrm{R} \%=-19,884+$ $\exp (8,400-3,739 \mathrm{Ds})\left(\mathrm{R}^{2}=0,97\right)$ estabelecidas para essas mesmas camadas, o valor de $\mathrm{S}=0,1 \mathrm{~cm}^{3} \mathrm{~cm}^{-3}$, considerado crítico (Greenland, 1981), não se constituiu condição mínima de aeração no Latossolo Roxo para o desenvolvimento radicular de milho.

De modo a relacionar $\mathrm{R} \%$, Ds e $\mathrm{S}$, a partir uma única equação estabeleceram-se as expressões: $\mathrm{R} \%=248,237-152,767 \mathrm{Ds}+174,124 \mathrm{~S}\left(\mathrm{R}^{2}=0,85\right)$ para a superficial e $\mathrm{R} \%=127,905-94,153 \mathrm{Ds}+78,069 \mathrm{~S}$ $\left(\mathrm{R}^{2}=0,94\right)$ para a subsuperficial. Tal relacionamento foi empregado também como classes de desenvolvimento radicular (Tabela 1), correspondentes a intervalos de densidade e macroporosidade do solo definidos pelos agrupamentos formados nos dendrogramas (Figs. 1 e 2).

Na Tabela 1, verifica-se que quando Ds $=1,0 \mathrm{~kg} \mathrm{dm}^{-3}$, na camada superficial, o valor de $\mathrm{R} \%$ será no máximo 63,5 do referencial (MAX-1) e quando Ds $=1,2 \mathrm{~kg} \mathrm{dm}^{-3}$, na subsuperficial, o valor de $\mathrm{R} \%$ será no máximo 48,0 . Tais diferenças de $\mathrm{R} \%$ sugerem que as condições estabelecidas para o tratamento de máxima produção limitaram o desenvolvimento radicular do milho nos tratamentos. As condições do solo no MAX-1, como homogeneidade e adequação das características físicas, químicas e morfoestruturais às necessidades da cultivar, explicam esta limitação.
$\mathrm{Na}$ Tabela 1, os valores de Ds e $\mathrm{S}$ relacionados ao valor mínimo de $\mathrm{R} \%$ (9,5 na camada superficial e 8,0 na subsuperficial) limitam a classe de desenvolvimento radicular "muito baixo" e correspondem ao valor de S considerado crítico por Greenland (1981). Nessa tabela, o valor crítico de $\mathrm{S}$ que restringe o desenvolvimento radicular do milho corresponde a $0,06 \mathrm{~cm}^{3} \mathrm{~cm}^{-3}$ na camada superficial e a $0,07 \mathrm{~cm}^{3} \mathrm{~cm}^{-3}$ na subsuperficial.

A distribuição dos valores médios de Ds e S no perfil do solo antes (AM) e depois da mobilização (DM) do solo é apresentada nas Figs. 3 e 4. Os valores médios observados incluem os obtidos nos tratamentos convencional (C) e de subsolagem (SC) por não diferirem estatisticamente entre si. A diferença entre AM e DM representa o efeito imediato das operações de preparo do solo para o plantio do milho no ano agrícola 1987/88.

Em AM, na camada superficial, os valores médios de Ds mantiveram-se em torno de $1,16 \mathrm{~kg} \mathrm{dm}^{-3} \mathrm{e}$ os de $\mathrm{S}$ em torno de $0,14 \mathrm{~cm}^{3} \mathrm{~cm}^{-3}$, enquanto em DM foram obtidos valores de Ds em torno de $1,13 \mathrm{~kg} \mathrm{dm}^{-3}$, e de $\mathrm{S}$ em torno de $0,19 \mathrm{~cm}^{3} \mathrm{~cm}^{-3}$. Por sua vez, as operações de preparo do solo diminuíram os valores de Ds e aumentaram os de $\mathrm{S}$, melhorando a porosidade do solo e aumentando o potencial de desenvolvimento radicular da cultivar, nessa camada (Tabela 1). Tal condição permaneceu inalterada por cerca de 26 dias no mês de novembro e parte de dezembro, com tendência a aumentar e retornando aos valores obtidos em AM em 18/01/88 (Fig. 5). Em conseqüência, a macroporosidade na camada superficial do solo e o potencial de desenvolvimento radicular diminuíram gradativamente durante o ciclo da cultura no ano agrícola 1987/88. Os efeitos das operações de preparo, nessa camada, nos tratamentos C e SC ocorreram por curto período, referendando os resultados obtidos por Corsini (1988), nesse mesmo solo.

Como pode ser observado nas Figs. 3 e 4, não ocorreu diferença nos valores de Ds e S entre AM e DM na camada subsuperficial. Os valores de S apresentaram uma maior variabilidade, principalmente no tratamento com subsolagem, justificada pela presença de trincas e fendas na estrutura da camada, em DM. Verifica-se ainda que os valores de Ds decrescem e os de $\mathrm{S}$ crescem à medida que se aprofunda no perfil do solo, característica da camada subsuperficial no Latossolo Roxo intensivamente 
TABELA 1. Classes de desenvolvimento radicular ( $R$ ) correspondentes a intervalos de densidade (Ds) e macroporosidade no solo.

\begin{tabular}{|c|c|c|c|c|c|c|}
\hline \multirow[t]{2}{*}{ Classe } & \multicolumn{3}{|c|}{ Camada superficial } & \multicolumn{3}{|c|}{ Camada subsuperficial } \\
\hline & $\mathrm{R}^{1}(\%)$ & Ds $\left(\mathrm{kg} \mathrm{dm}^{-3}\right)$ & $\mathrm{S}\left(\mathrm{cm}^{3} \mathrm{~cm}^{-3}\right)$ & $\mathrm{R}^{1}(\%)$ & Ds $\left(\mathrm{kg} \mathrm{dm}^{-3}\right)$ & $\mathrm{S}\left(\mathrm{cm}^{3} \mathrm{~cm}^{-3}\right)$ \\
\hline Muito alto & $>63,5$ & - & - & $>48,0$ & - & - \\
\hline Alto & $63,5-24,0$ & $1,00-1,14$ & $0,27-0,24$ & $48,0-34,0$ & $1,12-1,20$ & $-0,27-0,20$ \\
\hline Médio & $24,0-17,5$ & $1,14-1,27$ & $0,24-0,18$ & $34,0-17,0$ & $1,20-1,29$ & $0,20-0,15$ \\
\hline Muito Baixo & $17,5-9,5$ & $1,27-1,47$ & $0,18-0,10$ & $17,0-8,0$ & $1,29-1,34$ & $0,15-0,10$ \\
\hline Baixo & $9,5^{2}-3,0$ & $1,47^{2}-1,57$ & $0,10^{2}-0,06$ & $8,0^{2}-0,0$ & $1,34-1,40^{2}$ & $0,10^{2}-0,07$ \\
\hline Crítico & $<3,0$ & $>1,57$ & $<0,06$ & - & $>1,40$ & $<0,07$ \\
\hline
\end{tabular}

AX-1 e igual a 100.

2 Nível crítico estabelecido por Greenland (1981)

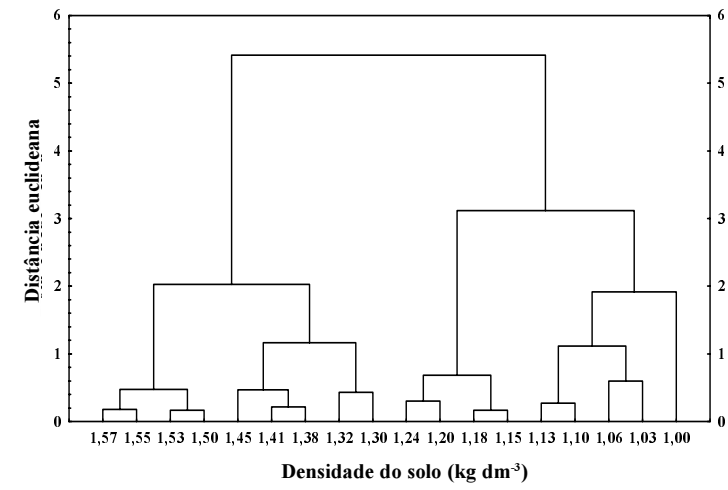

FIG. 1. Grupos selecionados segundo a porcentagem de raízes, densidade e macroporosidade do solo na camada superficial.

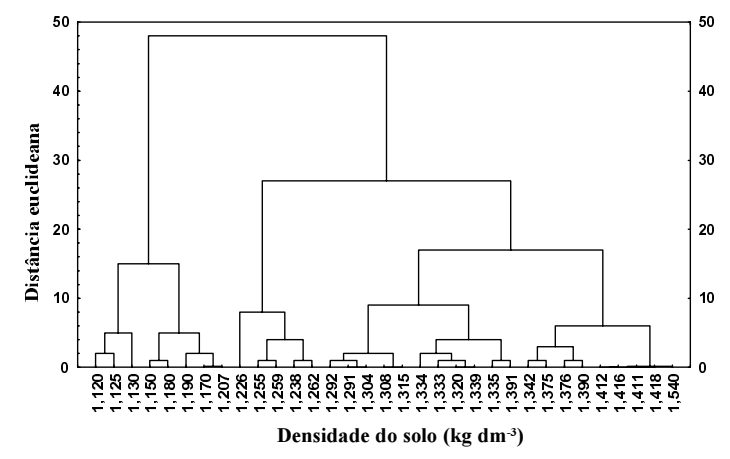

FIG. 2. Grupos selecionados segundo a porcentagem de raízes, densidade e macroporosidade do solo na camada subsuperficial.

cultivado. Na Fig. 5 observa-se que esses valores não se alteraram ao longo do ano agrícola 87/88.

O efeito residual dos sistemas de preparo do solo foi analisado pela variação de Ds e S, nos oito anos

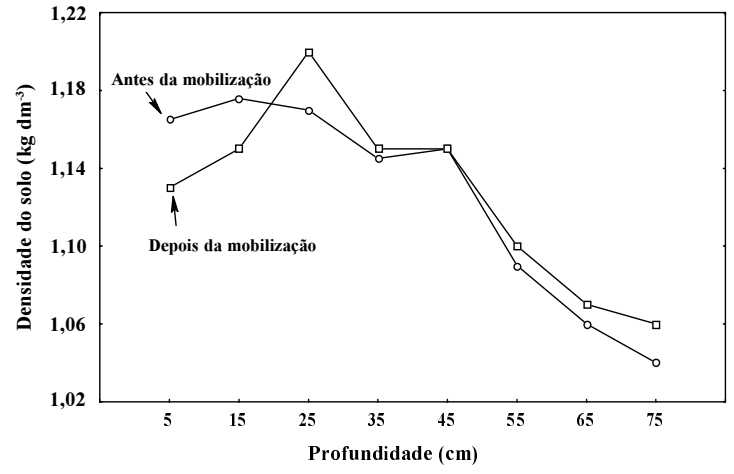

FIG. 3. Variação da densidade em função de profundidade do solo, antes e imediatamente após a instalação do experimento.

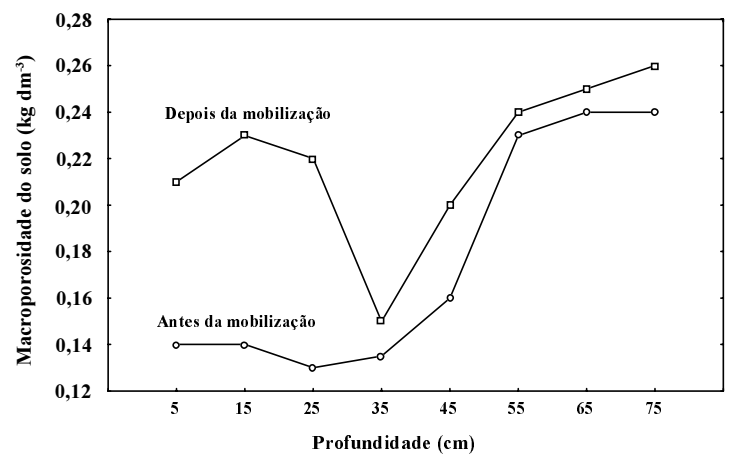

FIG. 4. Variação da porosidade de aeração em função da profundidade do solo, antes e imediatamente após a instalação do experimento.

agrícolas consecutivos em que se cultivou milho em sistema de plantio direto. Os valores $\mathrm{Ds}_{0}=1,165 \mathrm{~kg} \mathrm{dm}{ }^{-3} \mathrm{e}_{0}=0,140 \mathrm{~cm}^{3} \mathrm{~cm}^{-3}$, na camada superficial, e $\mathrm{Ds}_{0}=1,170 \mathrm{~kg} \mathrm{dm}^{-3} \mathrm{e}$ 


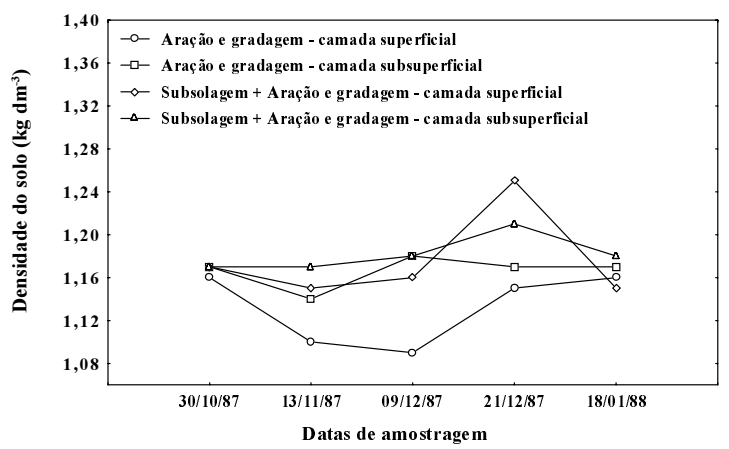

FIG. 5 . Variação da densidade do solo no ano agrícola $87 / 88$.

$\mathrm{S}_{0}=0,130 \mathrm{~cm}^{3} \mathrm{~cm}^{-3}$, na subsuperficial, na condição $\mathrm{AM}$, foram utilizados como referenciais por serem praticamente iguais à média dos valores encontrados ao término do período de pousio em cada ano agrícola estudado. Representam, dessa maneira, o solo estabilizado estruturalmente (Kay et al., 1988) e correspondem ao terceiro estádio do processo de degradação de solos cultivados intensivamente (Corsini, 1991). Assim, a variação de Ds e S em porcentagem, nas camadas amostradas ao longo dos anos foi estabelecida pelas relações $\Delta \mathrm{Ds}_{\mathrm{i}}=\mathrm{Ds}_{\mathrm{i}}-\mathrm{Ds}_{0}$ e $\mathrm{DS}_{\mathrm{i}}=\mathrm{S}_{\mathrm{i}}-\mathrm{S}_{0}$, para $\mathrm{i}=1,2, \ldots ., 8$, onde $\mathrm{Ds}_{\mathrm{i}}$ e $\mathrm{S}_{\mathrm{i}}$ são os valores médios de densidade do solo e macroporosidade a cada ano $\mathrm{i}$, a partir de 87/88.

Os valores de $\Delta \mathrm{Ds}_{\mathrm{i}} \mathrm{e} \Delta \mathrm{S}_{\mathrm{i}}$ obtidos na linha e entrelinha da cultura sob plantio direto estão apresentados nas Figs. 6 e 7. Nelas podem ser verificados que na camada superficial e na entrelinha os valores de $\Delta \mathrm{Ds}_{\mathrm{i}}$ aumentaram de $-3 \%$ a $7 \%$ entre $87 / 88$ e $91 / 92$, decrescendo de $7 \%$ a $0 \%$ entre $91 / 92$ e 94/95. O modelo $\mathrm{Ds}_{\mathrm{i}}=1,077+0,067 \mathrm{i}-0,007 \mathrm{i}^{2}(\mathrm{i}=1,2, \ldots, 8)$ com $\mathrm{R}^{2}=0,82$ representa a variação desse valor relativo ao longo dos anos agrícolas estudados. Nessa mesma camada e posição de amostragem, os valores de $\Delta \mathrm{S}_{\mathrm{i}}$ se comportaram de maneira inversa, decresceram de 0 a $-20 \%$ até o ano agrícola $89 / 90$, e cresceram nos anos seguintes (89/90 a 94/95), de $-20 \%$ a $70 \%$, numa tendência linear, conforme o modelo $\mathrm{S}_{\mathrm{i}}=0,077+0,026 \mathrm{i}$ $(i=3,4, \ldots, 8)$ com $R^{2}=0,93$. Os valores de $\Delta S_{i}$ começaram a aumentar dois anos antes de os valores de $\Delta \mathrm{Ds}_{\mathrm{i}}$ iniciarem a diminuir. Tal procedimento sugere que na camada superficial e na entrelinha da cultura os processos de microagregação e alterações estruturais de natureza biológica ocorrentes em solos mantidos sob plantio direto afetaram os valores de S mais intensamente que os de Ds (Monnier et al., 1973; Corsini, 1974; Pagliai et al., 1984). De 91/92 a 94/95, os valores de $\Delta \mathrm{S}_{\mathrm{i}}$ aumentaram e os $\Delta \mathrm{Ds}_{\mathrm{i}}$ decresceram na camada superficial com maior intensidade na linha da cultura. Os valores de $\Delta \mathrm{Dsi}$ decresceram em torno de $7 \%$ em EL e $16 \%$ em L. Por sua vez, os valores de $\Delta \mathrm{S}_{\mathrm{i}}$, tanto em EL como em $\mathrm{L}$, aumentaram em torno de $80 \%$. O aumento na porosidade estrutural no solo mantido sob plantio direto deveu-se, segundo Sidiras \& Pavan (1986) e Corsini (1993) à diminuição da repetibilidade no preparo do solo, à proteção representada pelos restos culturais de cada ano agrícola e ao aumento no teor de matéria orgânica na camada superficial. Nos dois primeiros casos, evita-se a ação da água da chuva sobre o solo exposto, principalmente sobre os agregados menores, menos estáveis. $\mathrm{O}$ aumento no teor de matéria orgânica aumenta a atividade biológica (Ehlers, 1975; Roth et al., 1986; Winter et al., 1990), possibilitando a formação de ácidos húmicos e de complexos argilo-orgânicos (Castro Filho, 1988), que diminuem a dispersibilidade da argila (Fuller et al., 1986; Brubaker et al., 1992; Fuller \& Goh, 1995) e possibilitam a formação de microagregados de maior estabilidade. Esse aumento foi mais efetivo na linha da cultura, em virtude das operações de plantio, pressões exercidas pelo sistema radicular e presença maior de raízes. De maneira geral, o aumento na porosidade estrutural na camada superficial do Latossolo Roxo tornou mais adequada a relação ar/água e o potencial de desenvolvimento radicular, como pode ser verificado na Tabela 1 .

Observa-se ainda (Fig. 6) que na camada subsuperficial os valores de $\Delta \mathrm{Ds}_{\mathrm{i}}$ na linha e entrelinha não sofreram alterações no solo sob plantio direto, ao contrário do ocorrido na camada superficial. A partir de 91/92, os valores de $\Delta \mathrm{Ds}_{\mathrm{i}}$ apresentaram leve tendência de aumento. Na Fig. 7, verifica-se que, nessa camada, os valores de $\Delta \mathrm{S}_{\mathrm{i}}$ mantiveram-se praticamente constantes no período $87 / 88$ a $91 / 92$, crescendo a seguir, principalmente na linha da cultura. A alteração na porosidade estrutural foi influenciada pelos mesmos fatores responsáveis pela alteração na camada superficial, porém em escala menor na entrelinha da cultura. 


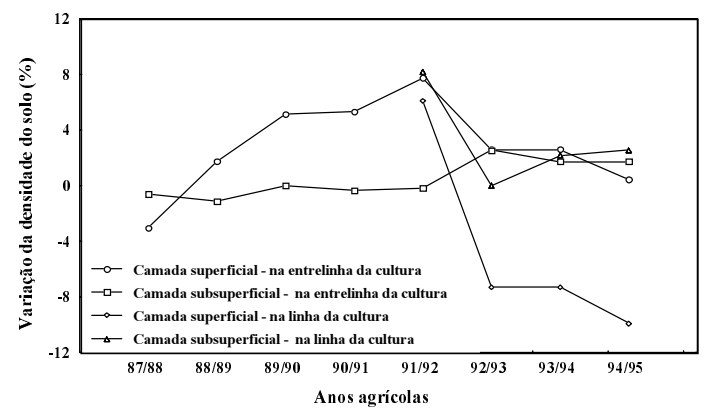

FIG. 6 Variação da densidade no solo ao longo dos anos em que ficou sob sistema de plantio direto de milho (1987 a 1995) .

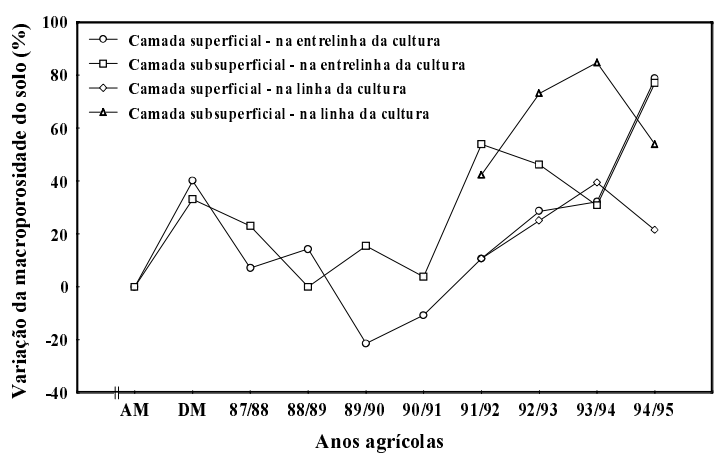

FIG. 7. Variação da porosidade de aeração no solo ao longo dos anos em que ficou sob sistema de plantio direto de milho (1987 a 1995).

As variações de Ds e S em razão da profundidade ao término do experimento são apresentadas nas Figs. 8 e 9. Pode-se observar que, nas camadas de 0 a $25 \mathrm{~cm}$ de profundidade, os valores de Ds, na linha da cultura e no solo sob plantio direto, foram superiores, e os de $\mathrm{S}$ inferiores aos encontrados no solo sob plantio convencional. Os valores observados na entrelinha da cultura no solo sob plantio direto se colocaram numa faixa intermediária. A partir dessa camada nenhuma alteração foi observada e os valores encontrados em AM e DM mantiveram-se constantes nos dois sistemas de cultivo. Verificou-se que a adoção do plantio direto por oito anos aumentou a macroporosidade nas camadas de $0-25 \mathrm{~cm}$ de profundidade do Latossolo Roxo, exceção feita à camada de 0-10 cm, na entrelinha da cultura, onde, devido ao efeito do tráfego de máquinas e pessoas, os valores de Ds e $\mathrm{S}$ não diferiram dos encontrados

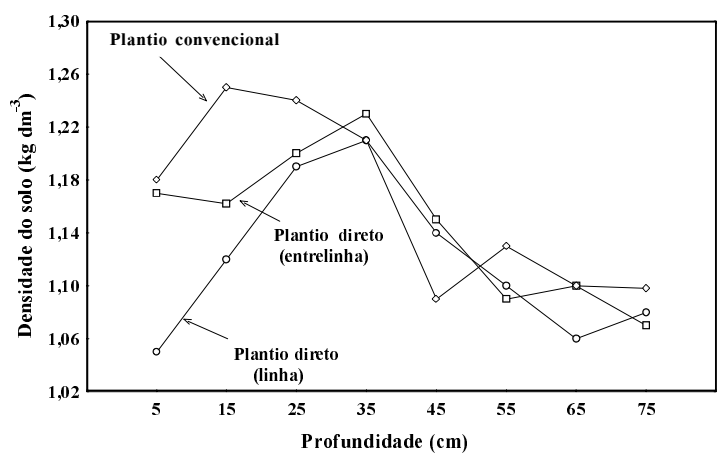

FIG. 8. Variação da densidade em função da profundidade do solo ao final do ano agrícola 94/95.

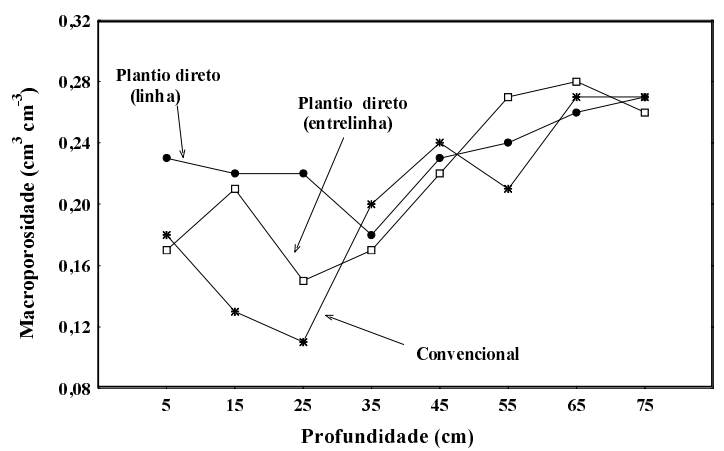

FIG. 9. Variação da macroporosidade em função da profundidade do solo ao final do ano agrícola 94/95.

no solo cultivado convencionalmente. Os valores de Ds e S na linha da cultura, ao término do experimento, nos tratamentos de plantio direto foram semelhantes aos obtidos em DM, sob os efeitos imediatos de $\mathrm{C}$ e SC.

\section{CONCLUSÕES}

1. A subsolagem com aração e gradagem aumenta a porosidade da camada superficial do solo, bem como o potencial de desenvolvimento radicular.

2. Nos três primeiros anos agrícolas, o sistema de plantio direto diminui a porosidade e o potencial de desenvolvimento radicular da camada superficial do solo; somente a partir do quinto ano agrícola é que esses parâmetros começam a crescer.

3. No sistema de plantio direto a recuperação da estrutura na camada superficial do Latossolo Roxo e na linha da cultura, para atingir níveis de densidade 
do solo, porosidade e níveis de desenvolvimento radicular semelhantes aos obtidos logo após a realização das operações mecânicas de preparo, inicia-se no quarto ano agrícola e completa-se no oitavo.

4. Outros fatores não relacionados à densidade $\mathrm{e}$ macroporosidade do Latossolo Roxo, na área experimental, limitam o desenvolvimento radicular em cerca de $37 \%$, na camada superficial, e $52 \%$ na subsuperficial.

\section{AGRADECIMENTOS}

Aos Engenheiros Agrônomos Marcelo Neves Machado e Paulo Henrique Trinque, pela colaboração na realização do experimento; à Fundação de Amparo à Pesquisa do Estado de São Paulo (FAPESP) e ao Conselho Nacional de Desenvolvimento Científico e Tecnológico (CNPq), pelas bolsas concedidas.

\section{REFERÊNCIAS}

ALZUBAIDI, A.; WEBSTER, G.R. Effect of tillage in combination with chemical amendments on reclamation of a Solonetic soils. Canadian Journal of Soil Science, Manitoba, v.62, p.641-649, 1982.

BLAKE, G.R. Bulk density. In: BLACK, C.A. (Ed.). Methods of soil analysis. Part.1. Physical and Mineralogical Properties. Madison: American Society of Agronomy, 1965. p.374-390. (Agronomy, 9)

BLEVINS, R.L.; THOMAS, G.W.; CORNELIUS, P.L. Influence of no-tillage and nitrogen fertilization on certain soil properties after 5 years of continuous corn. Agronomy Journal, Madison, v.60, n.3, p.383-386, 1977.

BLEVINS, R.L.; THOMAS, G.W.; CORNELIUS, P.L. Effect of lime application on no-tilled and conventionally tilled corn. Agronomy Journal, Madison, v.70, n.2, p.322-326, 1978.

BRUBAKER, S.C.; HOLZHLEY, C.S.; BRASHER, B.R. Estimating the water - dispersible clay content of soils. Soil Science Society of America. Journal, Madison, v.56, p.1227-1232, 1992.

CARTER, M.R.; KUNELIUS, H.T. Comparison of tillage and direct drilling for Italian ryegrass on the properties of a fine sandy loam soil. Canadian Journal of Soil Science, Manitoba, v.66, p.197207, 1986.
CARTER, M.R. Temporal variability of soil macroporosity on a fine sandy loam under mouldboard plouging and direct drilling. Soil and Tillage Research, Amsterdan, v.12, p.35-51, 1988.

CASAGRANDE, A.A.; FERREIRA, M.E.; CORSINI, P.C.; RODRIGUES, R. Associação entre sistemas de preparo do solo e fosfatagem em cana-de-açúcar (Saccharum spp.): efeitos no solo. Brasil Açucareiro, Piracicaba, v.97, n.4, p.48-62, 1981.

CASAGRANDE, A.A.; GODOY, A.; CORSINI, P.C. Efeito de sistemas mecânicos de cultivo nas propriedades físicas do solo num agrossistema açucareiro. Brasil Açucareiro, Piracicaba, v.4, p.30-49, 1975.

CASTRO FILHO, C. Effects of liming on characteristics of Brazilian Oxisol at three levels of organic matter as related to erosion. Ohio State University, 1988. 261p. Ph.D. Thesis.

CASTRO FILHO, C.; CORSINI, P.C.; SOARES, D.; POLITANO, W. Acceptance of soil and water conservation strategies and technologies in Southern Brazil. In: BAUM, E.; WOLFF, P.; ZOBISCH, M. A. (Eds.). Acceptance of soil and water conservation. Strategies and Technologies, v.3. Topics in applied resource management in the tropics. Witzenhausen: German Institute for Tropical and Subtropical Agriculture, 1993. p.341-363.

CHAN, K.Y.; MEAD, J.A. Surface physical properties of a sandy loam soil under different tillage practices.

Australian Journal of Soil Research, Melbourne, v.26, p.549-559, 1988.

CHAN, K.Y.; ROBERTS, W.P.; HEEMAN, O.F. Organic carbon and associated soil properties of a red earth after 10 years of rotation under different stubble and tillage practices. Australian Journal of Soil Research, Melbourne, v.30, p.71-83, 1992.

COOPER, A.W. Effects of tillage on soil compaction. In: AMERICAN SOCIETY OF AGRICULTURAL ENGINEERS. Compaction of agricultural soils. St. Joseph, MI: ASAE, 1971. p.315-364.

CORSINI, P.C. Impact of soil degradation on crop production in Brazil. Soil and Tillage Research, Amsterdan, v.20, p.253-263, 1991. 
CORSINI, P.C. Modificações de características físicohídricas em perfis das séries Jaboticabal e Santa Tereza, ocasionadas pelo cultivo intensivo. Científica, São Paulo, v.2, n.2, p.148-161, 1974.

CORSINI, P.C. Problemas causados pela compactação dos solos. STAB, Piracicaba, v.11, n.5, p.8-13, 1993.

CORSINI, P.C. Tillage practices of sugar-cane: effects on soil porosity and soil water retention. In: INTERNATIONAL CONFERENCE OF THE INTERNATIONAL SOIL TILLAGE RESEARCH ORGANIZATION, 11., 1988, Edinburgh. Proceedings... Edinburgh: Tillage and Traffic in Crop Production, 1988, v.2., p.619-624.

DALLA ROSA, A.D. Sistemas de cultivo e práticas mecânicas na recuperação de solos degradados. Porto Alegre: UFRGS, 1984. 19p. Tese de Doutorado.

DAVIDSON, J.M.; GRAM, F.; PINSON, D.I. Changes in organic matter and bulk density with depth under two cropping system. Agronomy Journal, v.59, p.375-378, 1977.

DEXTER, A.R. Advances in characterization of soil structure. Soil and Tillage Research, Amsterdan, v.11, p.199-238, 1988.

DOUGLAS, J.T. Macroporosity and permeability of some soil cores from England and France. Geoderma, Amsterdan, v.37, p.221-231, 1986.

EHLERS, W. Observations on earthworn channels and infiltration on tilled and untilled loss soil. Soil Science, v.119, p. 242-249, 1975.

EMBRAPA. Serviço Nacional de Levantamento e Conservação de Solos (Rio de Janeiro, RJ). Manual de métodos de análises de solo. Rio de Janeiro, 1979. 274 p.

FREEBAIRN, D.M.; LOCH, R.J.; COGLE, A.L. Tillage methods and soil water conservation in Australia. Soil and Tillage Research, Amsterdan, v.27, p.303$-325,1993$.

FULLER, L.G.; GOH, T.B.; OSCARSON, D.W. Cultivation effects on dispersible clay of soil aggregates. Canadian Journal of Soil Science, Manitoba, v.24, p.269-284, 1986.
FULLER, L.G.; GOH, T.B. Stability energy relationships and their application to aggregation studies. Canadian Journal of Soil Science, Manitoba, v.72, p.453-466, 1995.

GREENLAND, D.J. Soil management and soil degradation. Journal of Soil Science, London, v.31, p.301-322, 1981.

GUERIF, J. The influence of water-content gradient and structure anisotropy on soil compressibility. Journal of Agriculture Engineering Research, London, v.29, p.367-374, 1984.

HAMBLIN, A.P. The effect of tillage on soil physical properties. In: CORNISIER, P.S.; PRATLEY, J.E. (Eds.). Tillage - New Directions in Australian Agriculture. Melbourne: Australian Society of Agronomy, 1987. p.128-170.

JOHNSTON, J.R.; BROWNING, G.M.; RUSSEL, M.B. The effect of cropping practices on aggregation, organic matter content and lost of soil and water in the marshal. Soil Science Society of American. Proceedings, Madison, v.7, p.105-107, 1942.

KAY, B.D.; ANGERSI, D.A.; GROENEVELT, P.A.; BALDOCK, J.A. Quantifying the influence of cropping history on soil structure. Canadian Journal of Soil Science, Manitoba, v.68, p.359$368,1988$.

LAL, R. Tillage effects on soil degradation, soil resilience, soil quality, an sustainability. Soil and Tillage Research, Amsterdan, v.27, p.1-8, 1993.

MARQUES, J.Q.A. Política de conservação do solo. Rio de Janeiro: Ministério da Agricultura, 1949. 73p.

MONNIER, G.; STENGEL, P.; FIÉS, J.C. Une method de mesure de la densité apparente de petits agglomerats terreux: application à l'analyse des systems de porosité du sol. Annales Agronomiques, Paris, v.24, p. 533-545, 1973.

MUZZILI, O. Influência do sistema de plantio direto comparado ao convencional sobre a fertilidade da camada arável do solo. Revista Brasileira de Ciência do Solo, Campinas, v.7, n.1, p.95-102, 1983.

PAGLIARI, M.; LAMARCA, M.; LUCAMENTE, G.; GENOVESE, L. Effects of zero and conventional tillage on the length and irregularity of elongated pores in a clay loam under viticulture. Soil and Tillage Research, Amsterdan, v.4, p.433-444, 1984.

Pesq. agropec. bras., Brasília, v.34, n.2, p.289-298, fev. 1999 
PETERSEN, R.G.; CALVIN, L.I. Sampling. In: KLUTE, A. (Ed.). Methods of soil analysis. Part. 1: Physical and mineralogical methods. Madison: American Society of Agronomy, 1986. p.33-51.

PIDGEON, J.D.; SOANE, B.D. Effects of tillage and direct drilling on soil properties during the growing season in a long-term barley mono-culture system. Journal of Agricultural Science, Camberra, v.88, p.431442, 1977.

ROTH, C.H.; CASTRO FILHO, C.; MEDEIROS,G.B. Análise dos fatores físicos e químicos relacionados com a agregação de um Latossolo Roxo distrófico. Revista Brasileira de Ciência do Solo, Campinas, v.15, p.241-248, 1986.

SHEAR, G.M.; MOSCHER, W.W. Continuous corn by the no tillage and continue tillage methods a six-year comparison. Agronomy Journal, Madison, v.58, n.1, p.147-148, 1969.

SIDIRAS, N.; PAVAN, M.A. Influência do sistema de manejo do solo no nível de fertilidade. Revista Brasileira de Ciência do Solo, Campinas, v.10, p.163166, 1986.

SNEATH, P.H.A.; SOKAL, R.R. Numerical taxonomy. San Francisco: W.H. Freeman, 1973. 573p.

TARDIEU, F.; MANICHON, H. Caractérisation entant que capteur d'eau de l'enraciment du mais en parcelle cultiveé II. Une methode d'étude de la répartition verticale et horizontale des racines. Agronomie, Paris, v.6, n.5, p.415-425, 1986.
TENNANT, D. A test of a modified line intersect method of estimating root lenght. Journal Ecology, v.63, p.995-1001, 1975.

TRIPLETT JUNIOR, G.B.; VAN DOREN JUNIOR, D.M. Nitrogen phosphorus and potassium fertilization on no-tilled maize. Agronomy Journal, Madison, v.61, n.4, p.637-639, 1969.

TROUSE JUNIOR, A.C.; HUMBERT, R.P. Deep tillage in Hawaii I. Subsoiling. Soil Science, v.88, p.150$158,1959$.

VIEIRA, M.J. Propriedades físicas do solo. In: IAPAR. Plantio direto no estado do Paraná. Londrina, 1981. p.19-32. (Circular IAPAR, 23).

VIEIRA, M.J.; MUZZILI, O. Características físicas de um oxissolo sob diferentes sistemas de cultivo. Pesquisa Agropecuária Brasileira, Brasília, v.19, n.7, p.873-882, 1984.

VOMOCIL, J.A. Porosity. In: BLACK, C.A. (Ed.). Methods of soil analysis, Part1. Physical and Mineralogical Properties. Madison: American Society of Agronomy, 1965. p.299-314.

WINTER, J.P.; VORONEY, R.P.; AINSWORTH, D.A. Soil microarthropods in long-term no-tillage and conventional tillage corn production. Canadian Journal of Soil Science, Manitoba, v.70, p.641653,1990 . 